\title{
RX J0524+42: A New Asynchronous Magnetic CV
}

\author{
Robert Schwarz \\ Universitäts-Sternwarte, Geismarlandstr. 11, 37083 Göttingen, \\ Germany
}

\author{
A.D. Schwope, A. Staude, T. Urrutia \\ Astrophysikalisches Institut Potsdam, An der Sternwarte 16, D-14482 \\ Potsdam, Germany \\ A. Rau, G. Hasinger \\ Max-Planck Institut für extraterrestrische Physik, Postfach 1312, \\ D-85741 Garching, Germany
}

\begin{abstract}
Extensive optical photometry of the canditate magnetic CV RX J0524+42 has uncovered three persistent periods at 157, 146 and 136 minutes, which are the manifestation of the orbital motion of the system, the white dwarf spin and a possible side-band period of the latter. Depending on the interpretation of the 146 or the 136 minute period as the spin period, the system is an asynchronous by $7 \%$ or $14 \%\left(1-P_{\text {spin }} / P_{\text {orb }}\right)$, and therefore intermediate between the near-synchronous polars $(<2 \%)$ and the two DQ Her stars with long spin periods (EX Hya and V1025 Cen). Sampling the data over the beat period of 1.4 or 0.7 days reveals a prominent double-humped modulation on the spin period for certain fractions of the beat cycle. The phasing of this modulation strongly suggests that either pole switching onto two diametrically opposed accretion sites, or pole migration is operating in this system. With a period right within the period gap, RX J0524+42 is a key object for MCV evolution: it might be the first transition object between the DQ and AM Her subclasses where the white dwarf is currently in the process of synchronisation.
\end{abstract}

\section{Asynchronous magnetic CVs}

Until the turn of the millenium asynchronous MCVs basically fell into two distinct groups. The first group is the classical DQ Her stars (or intermediate polars) which have an asynchronism $\left(1-P_{\text {spin }} / P_{\text {orb }}\right)$ of $z 90 \%$, apart from the short-period systems EX Hya and V1025 Cen. Although some researchers prefer to subdivide this class into the more synchronous (EX Hya like) and the highly asynchronous (DQ Her like) systems, there is a general consensus that most of the DQ Her stars, (i) accrete via a disk (see V2400 Oph for the rare case of an diskless system; Hellier \& Beardmore 2002) and (ii) are in spin equilibrium. 
The second group with an asynchronism of $\lesssim 2 \%$ are the four asynchronous AM Her stars (or polars) V1500 Cyg, BY Cam, V1432 Aql and CD Ind, which are probably normal AM Hers desynchronised by a recent nova explosion. Contrary to the classical DQ Her stars these systems are believed to have accretion streams rather than disks, and are currently in the process of re-synchronisation. Potentially, the near-synchronous polars are key objects to identify possible modes of asynchronous accretion (pole switching, pole migration) and measure the synchronisation torques in MCVs. Observationally, the progress on these systems has been slow, mainly due to the requirement to monitor these system over the beat cycle of weeks and months as is evident from the mere single contribution to this conference (CD Ind, Cropper, Ramsay \& Marsh 2004). From an evolutionary point of view, Warner (2002) noted that the abundance of these systems (4 out 67 AM Hers) implies nova recurrence times of only $10^{3}$ years, which probably poses a problem for either the synchronisation timescales assumed or the assignment of all these systems as post-novae.

In the following we will present photometric data which identify the MCV RX J0524+42 as a possible transition object between the DQ Her and AM Her stars, thus making it the first 'true' intermediate polar. During this conference three other systems of possibly similar type have been put forward, V381 Vel (asynchronism 9\%; Tovmassian et al. 2004), HS0922 (asynchronism 13\%; Tovmassian et al. 2004) and V697 Sco (asynchronism 26\%; Woudt \& Warner 2004). At present none of these fulfills the 'Patterson criterion' for a credible asynchronous MCV (see Patterson 1994) of having two stable periods identified with $P_{\text {orb }}$ and $P_{\text {spin }}$, and should be therefore regarded as candidates only.

\section{Identification}

RX J0524+42 is a X-point source located at a distance of only $15^{\prime}$ east of the supernova remnant VRO 42.05.01 (Pineault et al. 1987) at the position $\alpha_{2000}=$ $05^{\mathrm{h}} 24^{\mathrm{m}} 30^{\mathrm{s}} 8$ and $\delta_{2000}=42^{\circ} 44^{\prime} 53^{\prime \prime}$ as measured in a later ROSAT-HRI pointing. First speculations that this point source might be an ejected pulsar associated with the SNR were rejected after spectroscopic identification of the bright $(V \sim$ $17^{\mathrm{m}}$ ) optical counterpart with a CV. Preliminary lists of ROSAT-discovered CVs also name this source 'Paloma', due to the obvious resemblance of the SNR to a pigeon. The optical spectrum has abundant emission lines of neutral $\mathrm{H}$ and $\mathrm{He}$ and higher excitation lines of the Bowen blend and HeII, which are tracers of magnetically channeled accretion. The flux of the HeII line is one third of that of the $\mathrm{H} \beta$ line. This is significantly lower compared to most AM Her stars or the prototypical asynchronous polar BY Cam, where it is of equal strength.

\section{Optical photometry}

Initial CCD photometry of RX J0524+42 was obtained on January 27 and 29 1992 with the $1.52 \mathrm{~m}$ telescope of the Observatoire de Haute-Provence. Between 1995 to $2001 \mathrm{RX}$ J0524+42 was observed for a total of 26 nights with the $70 \mathrm{~cm}$ telescope of the Astrophysical Institute Potsdam using a cryogenic cooled $1 \mathrm{x} 1 \mathrm{k}$ TEK-CCD. The large spacing between the individual nights caused strong aliasing effects. This was partly overcome with a dense monitoring of RX J0524+42 

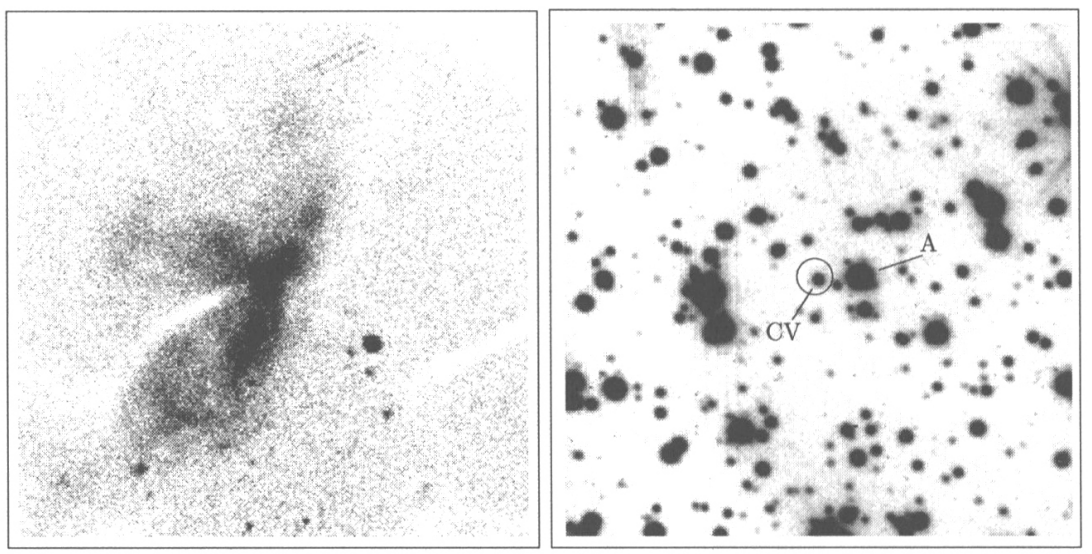

Figure 1. Left: ROSAT X-ray image of the Paloma SNR. The field of view is about $60 \times 60 \operatorname{arcmin}^{2}$ and the $\mathrm{CV}$ is the brightest point source $\sim 15$ arcmin west of the SNR. Right: $V$-band CCD image of RX J0524+42 obtained with the AIP $70 \mathrm{~cm}$ telescope. North is on top and east to the left. The size of the field is approximately $5 \times 5 \operatorname{arcmin}^{2}$. The circle refers to the $2 \sigma \mathrm{X}$-ray positional error. The position of the optical counterpart is $\alpha_{2000}=05^{\mathrm{h}} 24^{\mathrm{m}} 30^{\mathrm{s}} .4$ and $\delta_{2000}=42^{\circ} 44^{\prime} 51^{\prime \prime}$

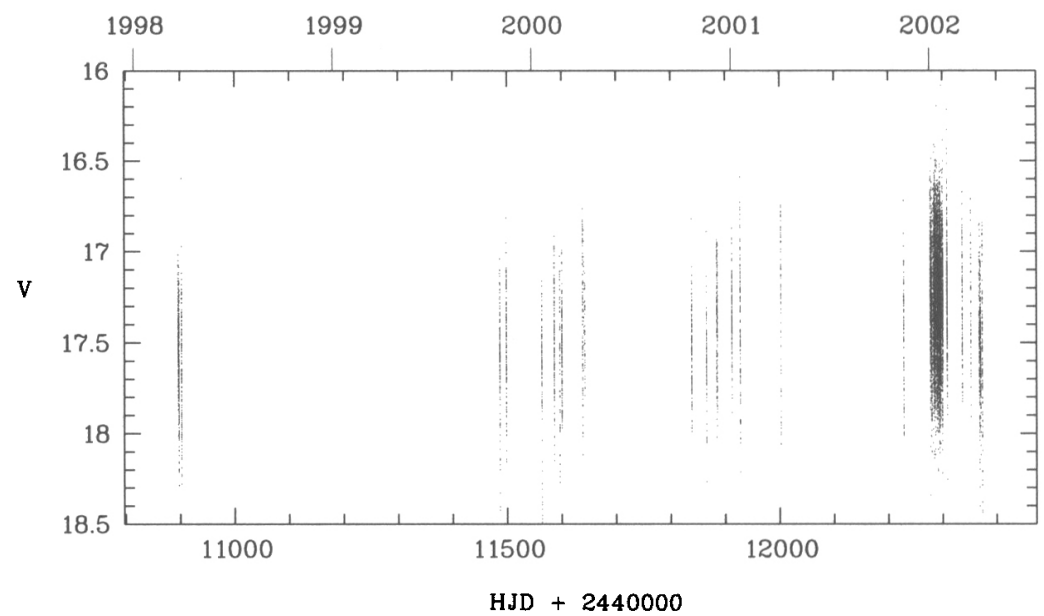

Figure 2. The long-term light curve of RX J0524+42.

during another 20 nights in 2002 January, with both the AIP $70 \mathrm{~cm}$ and the $1.23 \mathrm{~m}$ telescope at Calar Alto.

Some of the observations were affected by clouds and fog, but first order extinction correction was achieved by computing differential magnitudes with respect to star A (Figure 1). On two nights standard stars were observed from 


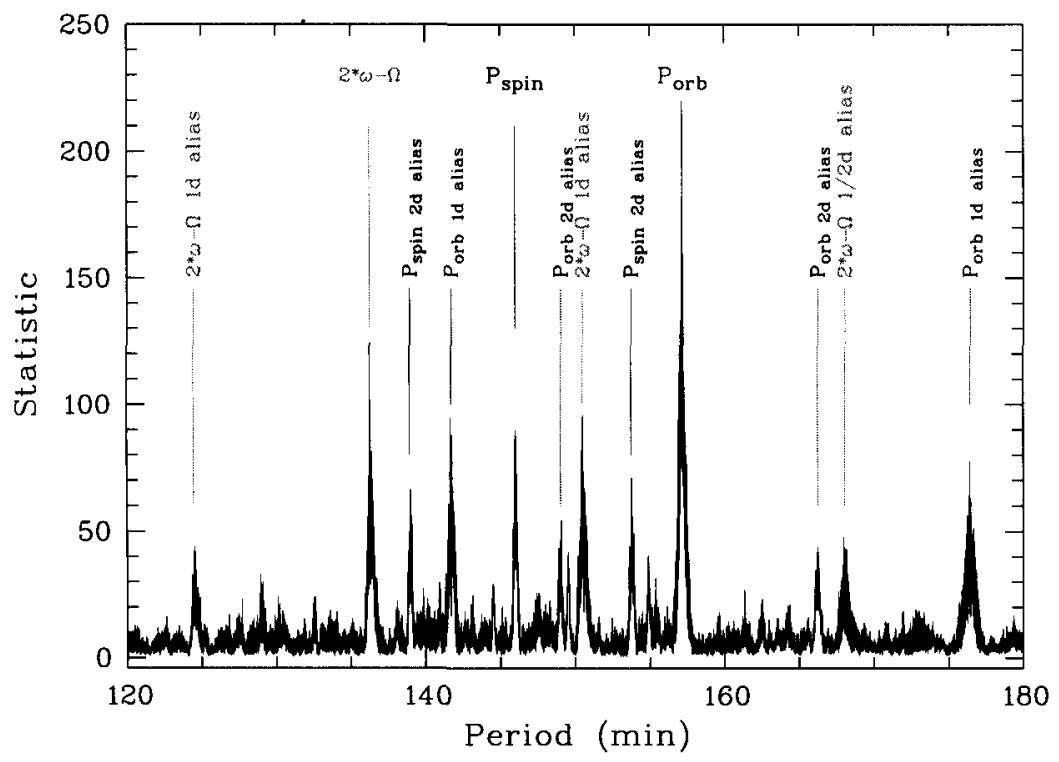

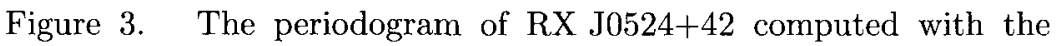
analysis-of-variance method from all available optical photometry. Periods identified correspond to case A with $P_{\text {spin }}=146 \mathrm{~min}$. For case B the spin period is at $136 \mathrm{~min}$ (the former $2 \omega-\Omega$ frequency), while the period at $146 \mathrm{~min}$ corresponds to the sub-harmonic of the side-band frequency $(\omega+\Omega)$.

which we derived a calibrated magnitude of star A of $V=13^{\mathrm{m}} 4$. The few data sets taken in $R$ and $I$ band or without a filter were calibrated using the same zero point, therefore ignoring possible colour terms. The long-term light curve plotted in Figure 2 shows the source at a constant mean orbital brightness level of $\mathrm{V} \sim 17^{\mathrm{m}} \cdot 5$ from 1998 to early 2002.

\subsection{Period analysis case A: $P_{\text {spin }}=146 \mathrm{~min}$}

We applied the analysis-of-variance method (AoV, Schwarzenberg-Cerny 1989) to the combined data set. The resulting periodogram (Figure 3 ) reveals a multitude of significant periods. In the range between $100-170 \mathrm{~min}$ we can identify three fundamental periods at 136, 146 and $157 \mathrm{~min}$, which are equidistant in frequency space, and must be therefore connected by a simple linear relation. If one assigns the longest of these as the orbital period $\left(P_{\text {orb }}=2 \pi / \Omega\right)$, and associates the period at $146 \mathrm{~min}$ with the spin period $\left(P_{\text {spin }}=2 \pi / \omega\right)$ the period at 136 min exactly represents the side-band frequency $2 \omega-\Omega$. All other main periods in the periodogram in that range then can be readily explained as one-day, two-day or half-day cycle count aliases of the fundamental periods. Of the three main signals, the orbital period is the strongest being a factor of 2 and 3 stronger than $P_{2 \omega-\Omega}$ and $P_{\text {spin }}$, respectively. This is similar to the power-spectrum of the asynchronous polar CD Ind (Ramsay et al. 1999), where also the orbital period 


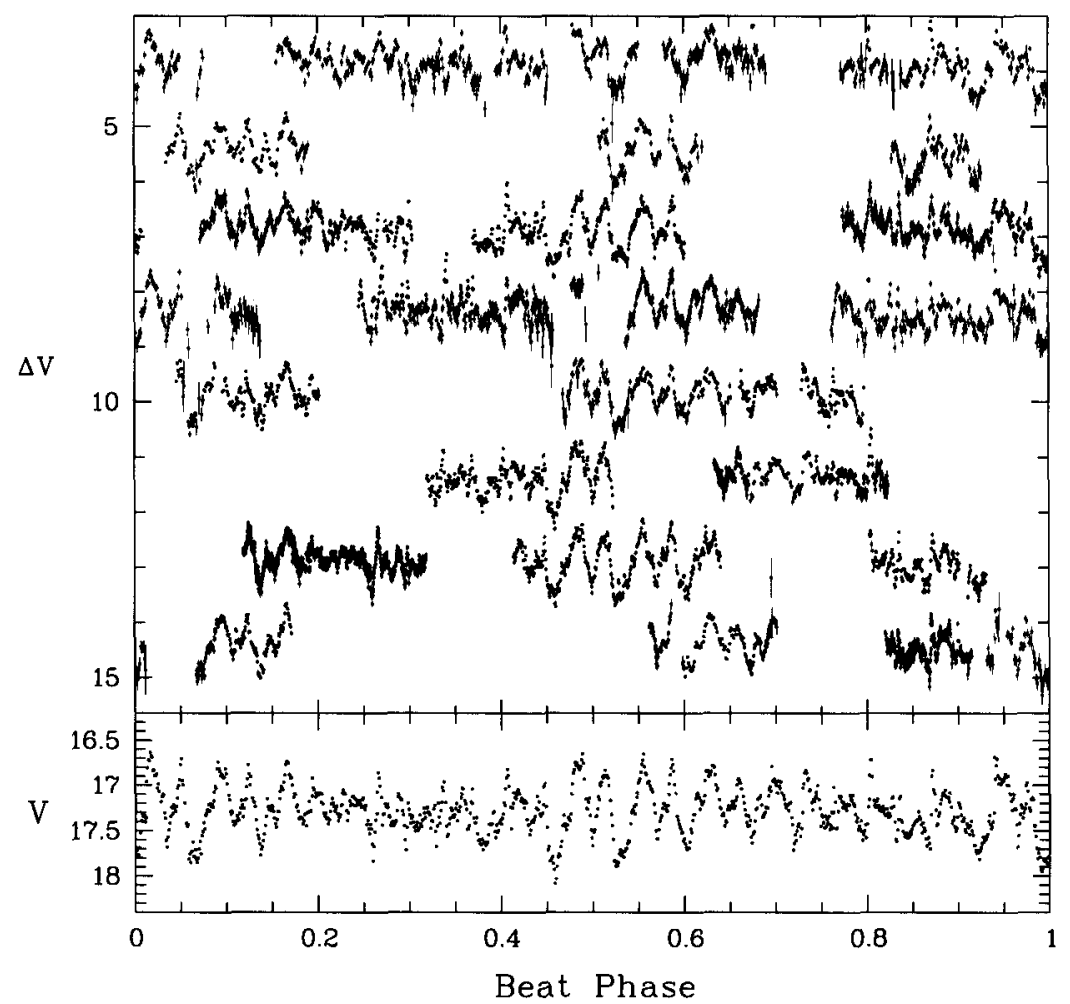

Figure 4. The collection of all optical data taken in 2002 January folded over the beat phase. In the lower panel the above data are phase averaged. Phase zero is arbitrarily defined at HJD 2450897.35376, the time of the deep optical minimum observed on March 24, 1998.

dominates, and $P_{2 \omega-\Omega}$ and $P_{\text {spin }}$ are of comparable strength. The presence of the side-band frequency $P_{2 \omega-\Omega}$, so far observed only in two other asynchronous polars (BY Cam; Silber et al. 1997 and CD Ind; Ramsay et al. 1999), was predicted for the power-spectra of disk-less asynchronous MCVs and can be taken as evidence for alternating accretion onto two diametrically opposed poles (Wynn \& King 1992). In addition a self-eclipsing geometry with $i+\beta>90^{\circ}$ is required for the detection of $P_{2 \omega-\Omega}$, a condition probably met in the case of RX J0524+42. The disk-less models of Wynn \& King (1992) also produce strong power at the orbital period, while the signal for $P_{\text {spin }}$ is absent in the case of a strict symmetry between the two poles. If we assume that the above interpretation is correct, the axis of the magnetic field will change its orientation to the mass donating star at the beat period $P_{\text {beat }}=\left(P_{\text {spin }}^{-1}-P_{\text {orb }}^{-1}\right)^{-1}$ of 1.41 days. A period close to the expected beat period is observed in the periodogram, but this might also fortuitously represent a sub-harmonic of the spin period $\left(14 \times P_{\text {spin }}\right)$. 


\begin{tabular}{|c|c|}
\hline \multirow[t]{2}{*}{ Period $(\min )$} & Signal \\
\hline & case $A$ case $B$ \\
\hline 157.1725 & $P_{\text {orb }}$ \\
\hline 145.9688 & $P_{(\omega+\Omega) / 2}$ \\
\hline 136.2561 & $P_{2 \omega-\Omega}$ \\
\hline 78.586 & $P_{\text {orb }} / 2$ \\
\hline 72.984 & $P_{\text {spin }} / 2 \quad P_{(\omega+\Omega)}$ \\
\hline 99.681 & $P_{\omega+\Omega / 2} \quad P_{\omega / 2+\Omega}$ \\
\hline 2043.576 & $2 P_{\text {beat }}$ \\
\hline
\end{tabular}

Table 1. Significant periods detected in the combined dataset of RX J0524+42

\subsection{Photometry around the beat cycle}

The rather short beat period of RX J0524+42 provides a good opportunity to study the photometric behaviour over the entire beat cycle, which is difficult to cover completely in the more synchronous polars. This is exemplified in Figure 4 for the 2002 January data set, which is the most homogenous and has the densest phase coverage. Surprisingly, individual light curves taken at a certain beat phase are very similar, even though the waveform constantly changes through the beat cycle and observations are weeks apart. There are marked stable patterns at two different beat phases $\left(\phi_{\text {beat }} \simeq 0.95\right.$ and $\left.\phi_{\text {beat }} \simeq 0.4\right)$, where a doublehumped light curve with an amplitude of $\sim 1.5$ mag is evident.

Such a waveform and amplitude in a MCV is likely to be caused by strong cyclotron beaming, and the phases of increased modulation correspond to a situation where matter is predominantly transferred onto one of the poles. The intervening intervals, when there is much less variation on the spin period, mark the phases where the pole-switch actually occurs. Rotating towards the infalling stream, the opposite pole can gradually receive more matter, and cyclotron beaming from both poles will mostly cancel out. Phases of maximum modulation are separated by $\Delta \phi_{\text {beat }} \sim 0.5$; thus pole-switching takes place after the magnetic axis of the white dwarf has changed its orientation by $180^{\circ}$ with respect to the infalling stream. Consequently, a highly symmetric geometry with two poles spaced apart by $180^{\circ}$ in longitude can explain the observed behaviour.

A confirmation of such view comes from the same data set, when folded over the white dwarf's spin period and sampled into individual beat phase intervals. The centroids of the double-humped modulation from two poles is displaced by 0.5 spin phases. This is equivalent to an azimuthal separation of $180^{\circ}$.

\subsection{Period analysis case B: $P_{\text {spin }}=136 \mathrm{~min}$}

The close resemblance of the spin-folded light curves from the two accretion spots for the above scenario, and the high degree of symmetry required, suggested the idea that there are possibly not two separate accretion spots but rather that the observed modulation comes from a single accretion region only. In that case, the 
beat frequency is only half than previously assumed $\left(P_{\text {beat }}=0.7\right.$ days $)$, while the shorter period of $136 \mathrm{~min}$ represents the white dwarf's spin. The middle period can then be explained as the first sub-harmonic of the $\omega+\Omega$ side-band frequency.

Light curves folded over the new spin period and selected for different beat phases show modulation from only one accretion spot, and a shift of the centroid of the bright phase indicates a migration of the visible accretion region by about $60^{\circ}$. The beat period of 0.7 days implied by this scenario is not detected in the periodogram. A firm decision about case A and B can probably be obtained only by future polarimetric measurements.

\section{Discussion}

The set of observations presented here certainly qualifies RX J0524+42 as a new asynchronous MCV with rather unique properties. The degree of asynchronism ( $7 \%$ or $14 \%$ ) detected in RX J0524+42 makes it the first possible transition object between the synchronous AM Her stars $(<2 \%)$ and the DQ Her star. The existence of such objects supports a possible evolutionary link, where the DQ Her stars are thought to be the progenitors of the AM Her stars at shorter periods (Patterson 1994, Cummings 2004).

Comparison of the synchronisation time scales $\tau_{\mathrm{syn}} \sim 10^{5}-10^{6} \mathrm{yr}$ with the lifetime of a CV in and above the period gap (a few $10^{8} \mathrm{yr}$ ) shows that the probability of detecting such object is rather low $\left(10^{-3}-10^{-2}\right)$ (Lamb 1995). Nevertheless, the finding of one such system out of $\sim 22$ long period DQ Her systems might still be consistent, if one allows fine tuning of the rather uncertain timescales. Confirmation of the evolutionary state, however, has to await the detection of a possible spin down of the white dwarf.

\section{References}

Cropper, M., Ramsay G. \& Marsh, T., 2004, these proceedings

Cummings A., 2004, these proceedings

Lamb D.Q., 1995, ASP Conf. Ser. 85, 519

Hellier C. \& Beardmore A.P., 2002, MNRAS 331, 407

Patterson J., 1994, PASP 106, 209

Pineault S., Landecker T. L. \& Routledge D., 1987, ApJ 315, 580

Ramsay G., Buckley D.A.H., Cropper M. \& Harrop-Allin M.K., 2001, MNRAS 303,96

Schwarzenberg-Czerny A., 1989, MNRAS 241, 153

Silber A.D., Szkody P., Hoard D.W. (+14 co-authors), 1997, MNRAS, 290, 25

Tovmassian G., Zharikov, S., Mennicket, R., Greiner, J., 2004, these proceedings Warner B., 2002, proceedings of 'Classical Nova Explosions', Sitges, Spain, AIP Conference Proceedings, Vol. 637. Sitges, Spain, 20-24 May, 2002, eds M. Hernanz and J. José, p. 3

Wynn G.A. \& King A.R., 1992, MNRAS 255, 83

Woudt P.A. \& Warner, B., 2004, these proceedings 